\title{
Erratum to: Matrix-Based Introduction to Multivariate Data Analysis
}

\section{Erratum to: \\ K. Adachi, Matrix-Based Introduction to Multivariate Data Analysis, https://doi.org/10.1007/978-981-10-2341-5}

In the original version of the book, belated corrections from author in Chapters 1,2 , $3,4,5,6,7,8,9,10,14$ and Appendix have to be incorporated. The erratum book has been updated with the changes.

\footnotetext{
The updated online version of this book can be found at https://doi.org/10.1007/978-981-10-2341-5_1 https://doi.org/10.1007/978-981-10-2341-5_2 https://doi.org/10.1007/978-981-10-2341-5_3 https://doi.org/10.1007/978-981-10-2341-5_4 https://doi.org/10.1007/978-981-10-2341-5_5 https://doi.org/10.1007/978-981-10-2341-5_6 https://doi.org/10.1007/978-981-10-2341-5_7 https://doi.org/10.1007/978-981-10-2341-5_8 https://doi.org/10.1007/978-981-10-2341-5_9 https://doi.org/10.1007/978-981-10-2341-5_10 https://doi.org/10.1007/978-981-10-2341-5_14 https://doi.org/10.1007/978-981-10-2341-5
} 